\title{
Rare KCNQ4 variants found in public databases underlie impaired channel activity that may contribute to hearing impairment
}

Jinsei Jung (1)', Haiyue Lin', Young Ik Koh², Kunhi Ryu ${ }^{3}$, Joon Suk Lee ${ }^{2}$, John Hoon Rim², Hye Ji Choi ${ }^{1}$, Hak Joon Lee', Hye-Youn Kim², Seyoung Yư ${ }^{2}$, Hyunsoo Jin ${ }^{4}$, Ji Hyun Lee $\mathbb{C}^{5}$, Min Goo Lee $\mathbb{C}^{2}$, Wan Namkung ${ }^{3}$, Jae Young Choi ${ }^{1}$ and Heon Yung Gee $\mathbb{i}^{2}$

\begin{abstract}
KCNQ4 is frequently mutated in autosomal dominant non-syndromic hearing loss (NSHL), a typically late-onset, initially high-frequency loss that progresses over time (DFNA2). Most KCNQ4 mutations linked to hearing loss are clustered around the pore region of the protein and lead to loss of KCNQ4-mediated potassium currents. To understand the contribution of KCNQ4 variants to NSHL, we surveyed public databases and found 17 loss-of-function and six missense KCNQ4 variants affecting amino acids around the pore region. The missense variants have not been reported as pathogenic and are present at a low frequency (minor allele frequency $<0.0005$ ) in the population. We examined the functional impact of these variants, which, interestingly, induced a reduction in potassium channel activity without altering expression or trafficking of the channel protein, being functionally similar to DFNA2-associated KCNQ4 mutations. Therefore, these variants may be risk factors for late-onset hearing loss, and individuals harboring any one of these variants may develop hearing loss during adulthood. Reduced channel activity could be rescued by KCNQ activators, suggesting the possibility of medical intervention. These findings indicate that KCNQ4 variants may contribute more to late-onset NSHL than expected, and therefore, genetic screening for this gene is important for the prevention and treatment of NSHL.
\end{abstract}

\section{Introduction}

KCNQ4 (KV7.4), a voltage-gated potassium channel, plays a critical role in the auditory function of the inner ear by regulating $\mathrm{K}^{+}$recycling and homeostasis, together with other $\mathrm{K}^{+}$ion channels (KCNQ1/KCNE1), gap junctions (GJB2, GJB3, and GJB6), and transporters $\left(\mathrm{Na}^{+} /\right.$ $\left.\mathrm{K}^{+} / 2 \mathrm{Cl}^{-}\right)^{1,2}$. The KCNQ4 gene was first cloned and

\footnotetext{
Correspondence: Heon Yung Gee (hygee@yuhs.ac.kr) or Jae Young Choi (jychoi@yuhs.ac)

${ }^{1}$ Department of Otorhinolaryngology, Brain Korea 21 PLUS Project for Medical Sciences, Yonsei University College of Medicine, Seoul 03722, Korea 2Department of Pharmacology, Brain Korea 21 PLUS Project for Medical Sciences, Yonsei University College of Medicine, Seoul 03722, Korea Full list of author information is available at the end of the article. These authors contributed equally: Jinsei Jung, Haiyue Lin, Young Ik Koh
}

characterized by Kubisch et al. ${ }^{3}$. They mapped it to the DFNA2 (autosomal dominant non-syndromic hearing loss; ADNSHL) locus, and a dominant-negative mutation was identified in the DFNA2 pedigree. DFNA2 is characterized as a late-onset disease, with high-frequency hearing loss that progresses to all frequencies over time ${ }^{3,4}$.

KCNQ4 is one of the most commonly mutated genes in $\mathrm{ADNSHL}^{5}$. Approximately 30 pathologic mutations in KCNQ4 have been identified as the cause of DFNA2 (www.deafnessvariationdatabase.org or www.hgmd.cf.ac. uk/ac/index.php). The KCNQ4 channel consists of six transmembrane domains, a pore region, and two intracellular termini ${ }^{6}$. The mutation hotspots in KCNQ4 associated with DFNA2 are clustered around the pore 
region ${ }^{7}$. The first described missense mutation (p.G285S ${ }^{3}$ as well as p.L274S, p.W276S, p.L281S, p.G285C, and p. G296S, which correspond to changes around the pore region of KCNQ4, induced a loss of channel function or decreased membrane expression of the channel protein $^{4,8-15}$.

Age-related hearing loss (ARHL) is the most common sensory deficit among the elderly and progresses slowly, similar to DFNA2. ARHL is a complex disease resulting from an interplay between genetic and environmental factors ${ }^{16}$. However, the contribution of genetic predisposition to ARHL is not clear and, therefore, often underestimated. Several single nucleotide polymorphisms in KCNQ4 are significantly associated with $\mathrm{ARHL}^{17}$, and it is plausible that the hypomorphic mutations in $K C N Q 4$ might contribute to $\mathrm{ARHL}^{18}$. In addition, $K C N Q 4$ variants are also implicated in noiseinduced hearing loss ${ }^{19}$.

In this study, we examined $K C N Q 4$ variants in public databases and found several missense variants around the pore region of KCNQ4 that are present at a low frequency in the general population and have not been associated with DFNA2. As the pathogenicity or clinical significance of these variants is unknown, we investigated the effects of these variants on KCNQ4 function and found that they significantly decreased $\mathrm{K}^{+}$channel activity. These findings suggest that these $\mathrm{KCNQ} 4$ variants may contribute to NSHL or ARHL.

\section{Materials and methods KCNQ4 variants}

KCNQ4 variants were examined in several databases: HGMD Professional (http://www.hgmd.cf.ac.uk/ac/index. php), ClinVar (https://www.ncbi.nlm.nih.gov/clinvar/), the Deafness Variation Database (http://www. deafnessvariationdatabase.org/), and the Genome Aggregation Database (gnomAD) (http://gnomad. broadinstitute.org/). A whole-genome sequencing dataset of 397 Korean individuals from the National Biobank of Korea of the Centers for Disease Control and Prevention was also used.

\section{Plasmid construction and site-directed mutagenesis}

Complementary DNAs (cDNAs) of human KCNQ4 were purchased from OriGene Technologies (Rockville, MD, USA) and subcloned into the pENTR-D-TOPO vector (Invitrogen, Carlsbad, CA, USA). Expression vectors were constructed using LR clonase (Invitrogen) following the manufacturer's instructions, and a Myg- or FLAG-tag was inserted in the $\mathrm{N}$-terminus. $\mathrm{KCNQ} 4$ variant clones were generated by PCR-based site-directed mutagenesis using the Quick Change II XL Site-Directed Mutagenesis Kit (Agilent Technologies, Santa Clara, CA, USA).

\section{Cell culture and transfection}

Human embryonic kidney 293 (HEK 293) and Chinese hamster ovary $(\mathrm{CHO})$ cells were cultured in Dulbecco's modified essential medium and RPMI 1640 medium, respectively, supplemented with $10 \%$ fetal bovine serum and penicillin $(50 \mathrm{IU} / \mathrm{mL}) /$ streptomycin $(50 \mu \mathrm{g} / \mathrm{mL})$ (Invitrogen). Cells were transfected with wild-type (WT) or mutant KCNQ4 plasmids using Lipofectamine and PLUS reagent, or Turbofect (Thermo Fisher Scientific, Waltham, MA, USA), according to the manufacturer's instructions. For electrophysiological experiments, $\mathrm{CHO}$ K1 cells were cotransfected with $0.9 \mu \mathrm{g}$ of the human $\mathrm{KCNQ} 4$ plasmids with $0.1 \mu \mathrm{g}$ of a green fluorescent protein (GFP) gene-containing expression plasmid to visualize the transfected cells. Experiments were performed within $24-36 \mathrm{~h}$ after transfection.

\section{Immunoblotting, immunoprecipitation, surface \\ biotinylation, and immunofluorescence}

Experiments were performed as described previously ${ }^{20}$. Anti-BiP (ab21685), anti- $\beta$-actin (ab6276, Abcam, Cambridge, UK), anti-Myc (sc-40), anti-aldolase A1 (sc-12059, Santa Cruz Biotechnology, Dallas, TX, USA), antiGOLGB1 (HPA011008), and anti-FLAG (F3165, Sigma-Aldrich, St. Louis, MI, USA) antibodies were purchased from commercial sources. Coimmunoprecipitation was performed using EZview Red Anti-FLAG M2 and anti-c-Myc Affinity Gel (Sigma). Surface biotinylation was performed using $0.3 \mathrm{mg} / \mathrm{mL}$ EZ-Link Sulfo-NHS-SSBiotin and NeutrAvidin (Thermo Fisher Scientific). Immunoblotting was performed using primary antibodies at a 1:1000 dilution, followed by corresponding antiisotype secondary antibodies (Santa Cruz Biotechnology) at a 1:2000 dilution. Signals were visualized using the SuperSignal West-Pico Kit (Thermo Fisher Scientific). For immunofluorescence, blocking buffer containing $10 \%$ donkey serum and $1 \%$ bovine serum albumin in phosphate buffered saline was used, and dilutions of primary and fluorophore-tagged secondary antibodies were 1:100 and 1:2000, respectively. Confocal images were obtained with a Carl Zeiss LSM780 instrument; ZEN software was used for image processing.

\section{Electrophysiology assay}

Whole-cell patch clamp techniques were used for measuring channel activity in KCNQ4-expressing CHO$\mathrm{K} 1$ cells. Cells were transferred to a bath mounted on the stage of an IX-71 inverted microscope (Olympus, Osaka, Japan) equipped with a light source set to green fluorescence excitation wavelengths. Patch clamp experiments were performed at room temperature $\left(23^{\circ} \mathrm{C}-25^{\circ} \mathrm{C}\right)$. Microglass pipettes (World Precision Instruments, Sarasota, FL, USA) were fabricated using a PC-10 dual-stage glass micropipette puller (Narishige, Tokyo, Japan) and an 
MF-830 microforge with a resistance of 2.0-2.5 $\mathrm{M} \Omega$ (Narishige, Tokyo, Japan). Currents were recorded using an Axopatch 200B amplifier and Digidata $1440 \mathrm{~A}$ interface, digitized at $10 \mathrm{kHz}$ and low pass-filtered at $5 \mathrm{kHz}$ by pClamp software v. 10.3 (Molecular Devices, Sunnyvale, CA, USA). The series resistance was compensated by an offset circuit in Axopatch 200B. All voltage and current trace data were analyzed using Clampfit v. 10.3 and Origin v. 8.0 software (Microcal, Northampton, MA, USA). The step pulse protocol used for KCNQ4 channel recordings started from a holding potential of $-80 \mathrm{mV}$, depolarized for $2 \mathrm{~s}$ from $-80 \mathrm{mV}$ to $20 \mathrm{mV}$ with $20 \mathrm{mV}$ increments, and followed by a tail pulse at $-50 \mathrm{mV}$ for $0.5 \mathrm{~s}$. Wholecell patch clamp analysis was conducted using a bath solution containing $147 \mathrm{mM} \mathrm{NaCl}, 5 \mathrm{mM} \mathrm{KCl}, 1.5 \mathrm{mM}$ $\mathrm{CaCl}_{2}, 1 \mathrm{mM} \mathrm{MgCl}_{2}, 10 \mathrm{mM}$ HEPES, and $10 \mathrm{mM}$ glucose (adjusted to $\mathrm{pH} 7.4$ with $\mathrm{NaOH}$ ). The pipette solution contained $130 \mathrm{mM} \mathrm{KCl}, 10 \mathrm{mM}$ HEPES, $1 \mathrm{mM} \mathrm{CaCl}$, $10 \mathrm{mM}$ EGTA, and $3 \mathrm{mM} \mathrm{Mg-ATP}$ (adjusted to $\mathrm{pH} 7.2$ with $\mathrm{KOH})$.

\section{Fluorescence-based thallium flux assay}

CHO-K1 cells were transfected in 96-well plates with KCNQ4 variants or empty plasmids. After $48 \mathrm{~h}$, the medium was replaced with $80 \mu \mathrm{L} /$ well of FluxOR (Invitrogen) loading buffer and incubated for $1 \mathrm{~h}$ at $37^{\circ} \mathrm{C}$ in the dark. The loading buffer was removed, and $100 \mu \mathrm{L}$ of assay buffer was added to each well. To activate the KCNQ4 channels, cells were pretreated with $10 \mu \mathrm{M}$ retigabine for $10 \mathrm{~min}$. FluxOR fluorescence (excitation/ emission: $490 / 525 \mathrm{~nm}$ ) was recorded $10 \mathrm{~s}$ prior to the addition of $20 \mu \mathrm{L}$ of the stimulus buffer containing a low level of thallium ions, and the fluorescence was monitored for an additional $15 \mathrm{~s}$. FluxOR fluorescence was recorded using a Zyla sCMOS camera (Andor Technology) and a Nikon Eclipse Ti inverted microscope (Nikon Instruments) and analyzed using Metamorph analysis software (Molecular Device). All buffers were prepared according to the manufacturer's instructions.

\section{Three-dimensional structure modeling}

To examine the effects of the variants at the protein level, structural modeling of KCNQ4 was performed using Swiss-Model (https://swissmodel.expasy.org/). Amino acid residues surrounding the pore region (residues 270-345) of KCNQ1 of Xenopus laevis (PDB 5vms), which shares $70 \%$ sequence identity with human KCNQ4 (resides 256-326), was used as the template structure. The tertiary structure of the domains was generated, and the 3D structures of the normal and mutated proteins were visualized using the UCSF Chimera package (http:// www.cgl.ucsf.edu/chimera). Protein stability was predicted using Site-Directed Mutator (http://marid.bioc. cam.ac.uk/sdm2/), I-Mutant Suite (http://gpcr2.biocomp.
unibo.it/cgi/predictors/I-Mutant3.0/I-Mutant3.0.cgi), and CUPSAT (http://cupsat.tu-bs.de/).

\section{Results \\ DFNA2-associated KCNQ4 variants}

In HGMD Professional, $36 \mathrm{KCNQ4}$ variants have been reported, with most of them linked to ADNSHL except the c.1525 G > T;p.E509* variant, which is associated with autism spectrum disorder (Fig. 1). We reported three pathogenic variants, including one founder mutation, in Koreans (Fig. 1b) ${ }^{21,22}$. Among the pathogenic variants of KCNQ4, there are also three in-frame deletions (Fig. 1b). In addition, c. $648 \mathrm{C}>\mathrm{T}$ is a synonymous variant but was detected in an individual with DFNA2.

In ClinVar, $106 \mathrm{KCNQ} 4$ variants have been reported, 24 of which are also found in HGMD Professional. Of the 82 KCNQ4 variants reported only in ClinVar, 70 are single nucleotide changes, and 12 are copy number variations (CNVs) that span large genomic regions affecting multiple genes, including $K C N Q 4$. Of the 70 single nucleotide variants, five are pathogenic or likely pathogenic, 35 are benign or likely benign, and 30 are of uncertain significance. Of the 12 CNVs in ClinVar, nine are pathogenic, and three are of uncertain significance. Nine pathogenic CNVs, including $K C N Q 4$, are mostly linked to severe phenotypes, such as global developmental delay, intellectual disability, and cardiac defects. One CNV, a duplication of chr1:849,467-248,224,649 (hg19), is associated with ear anomaly, short stature, cardiovascular anomaly, and polydactyly.

\section{KCNQ4 variants in the general population}

The gnomAD currently contains 123,136 exome sequences and 15,496 whole-genome sequences from unrelated individuals. There are $1110 \mathrm{KCNQ} 4$ variants in gnomAD, of which 297 are missense or loss-of-function (LoF) variants. Of the $19 \mathrm{LoF}$ KCNQ4 variants, 15 are nonsense or frameshift, and four affect an obligatory splice site (Table 1). The minor allele frequency (MAF) of these LoF alleles is very low (less than 0.0003234). None of them has been reported in HGMD Professional or ClinVar, and their pathogenicity or clinical significance is unknown. However, given that c.211delC;p.Q71Sfs"68 and c.725 G > A;p.W242* are reported as pathogenic in HGMD Professional, seven LoF variants (p.H77Lfs*159, p. R95P*44, p.H102 Tfs*37, p.Q122*, c.405+2T>G, p.Y160*, and p.Y232*) might be pathogenic, but this needs to be experimentally demonstrated.

Of the 278 missense $K C N Q 4$ variants in gnomAD, we examined the changing amino acid residues between the transmembrane domains (TM) 5 and 6 (aa 259-296) surrounding the pore region (aa 271-292), as most known KCNQ4 variants linked to hearing loss are clustered around this region ${ }^{7}$. There are six variants in this region of 


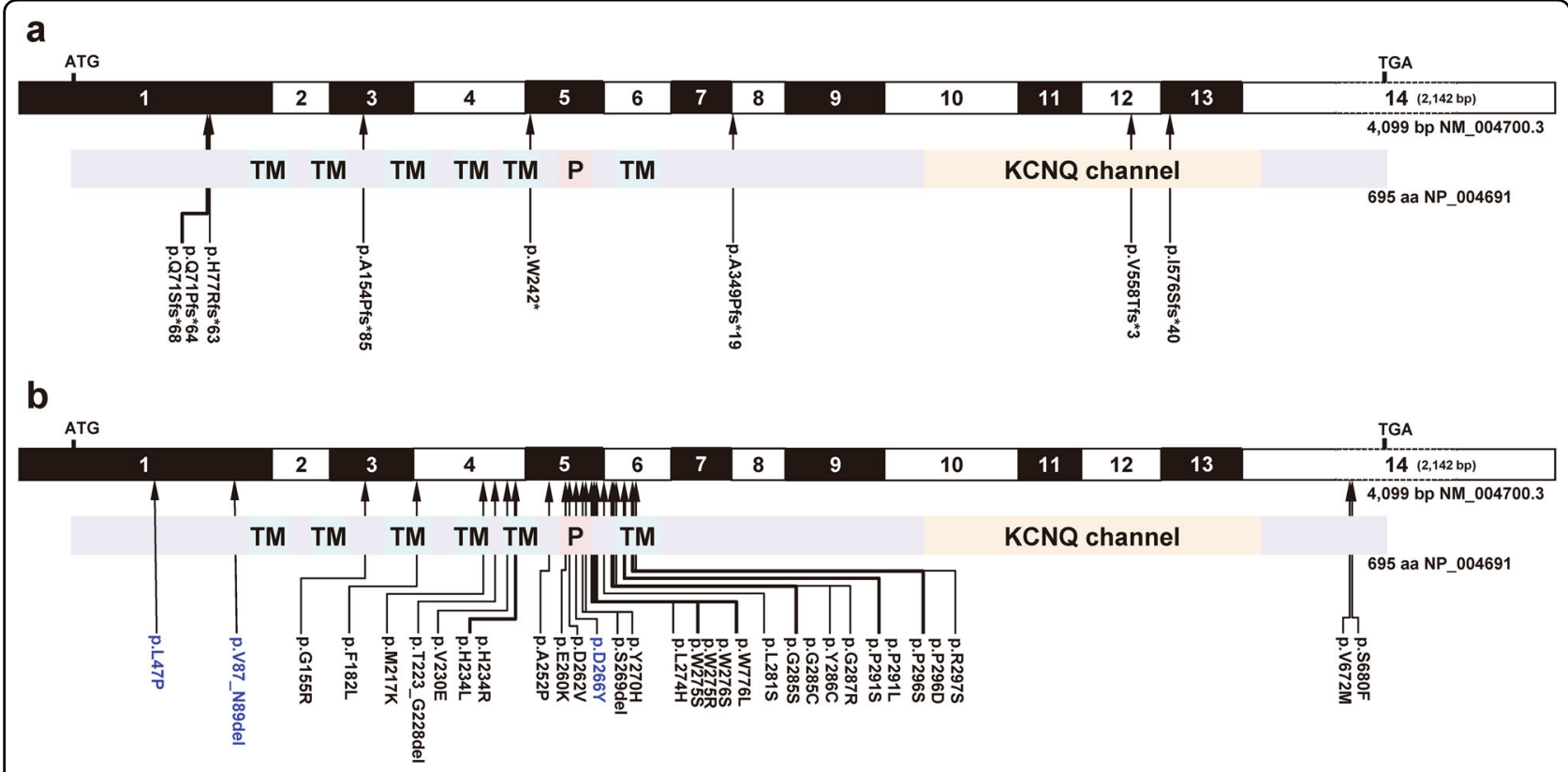

Fig. 1 Autosomal dominant non-syndromic hearing loss (DFNA2)-associated KCNQ4 variants. a Exon structure of human KCNQ4 CDNA and domain structure of KCNQ4 are shown. Only variants identified in individuals with DFNA2 are shown. KCNQ4 contains 14 exons; the positions of the start (ATG) and stop (TGA) codons are indicated. Loss-of-function mutations, such as nonsense and frameshift mutations, are indicated. $\mathbf{b}$ Missense and in-frame deletion mutations are indicated. Note that mutations are clustered in exons 5 and 6 , which encode part of the transmembrane (TM) 5 , pore region, and TM6. The pathogenic variants identified by our group are shown in blue

KCNQ4 in gnomAD, and none of them has been reported in either HGMD Professional or ClinVar except p.T278A, which has been reported in ClinVar as of unknown significance (Table 2 and Supplementary Fig. 1). These variants are rare, and their MAFs are less than 0.00003 . In addition, their pathogenicity or clinical significance is unknown.

We also examined a whole-genome sequencing dataset of 397 Korean individuals and found six missense KCNQ4 variants, but none of them corresponded to the pore region of the protein (Supplementary Table 1).

\section{Functional effects of KCNQ4 variants around the pore region}

As the effects on KCNQ4 function of the six missense variants around the pore region reported in gnomAD have not been investigated, we aimed to characterize these variants and a missense variant (p.R433W) detected in the Korean dataset.

We first performed immunofluorescence and surface biotinylation assays to examine the expression levels of the WT and variant KCNQ4 proteins at the plasma membrane in HEK 293 cells. Using immunofluorescence with endoplasmic reticulum and Golgi markers, the WT and all variant $\mathrm{KCNQ} 4$ proteins were detected at the cell surface (Fig. 2a). Surface biotinylation assays also confirmed that all variant proteins reached the plasma membrane (Fig. 2b). The expression levels of variant proteins were comparable to that of WT KCNQ4 even when overexpressed (Fig. 2b).

Because the KCNQ4 channel functions as a homo or heterotetramer of subunits ${ }^{10}$, we examined whether variant KCNQ4 proteins affected heteromeric assembly with the WT protein. Coimmunoprecipitation demonstrated that variant proteins were able to interact with the WT protein, indicating that heterotetramers containing the WT and variant proteins can form (Fig. 2c). Taken together, these results demonstrate that variant proteins do not exhibit defects in trafficking or subunit assembly.

To determine the effects of KCNQ4 variants on channel function, whole-cell currents were recorded in CHO-K1 cells transfected with WT or variant $K C N Q 4$ clones. The p.W276S mutation, which is known to cause DFNA2 ${ }^{3}$, was used as a positive control ${ }^{23}$, and a common single nucleotide polymorphism ( $\mathrm{rs} 34286752, \mathrm{c} .1365 \mathrm{~T}>\mathrm{G}$; $\mathrm{p}$. $\mathrm{H} 455 \mathrm{Q}$ ), the MAF of which is 0.2584 , was also included in the functional study. All variants showed reduced potassium channel activity (Fig. 3a, b and Supplementary Fig. 1). Pore region variants p.T278A, p.S273A, p.L281M, and p.L295P as well as p.R433W produced currents of $\sim 40-53 \%$ of WT levels (Fig. 3c and Supplementary Fig. 2). In addition, p.N264S and p.S269F showed no channel function as they produced basal level currents similar to those recorded in GFP- or p.W276S-transfected CHO cells (Fig. 3a-c and Supplementary Fig. 2). Because DFNA2-associated $K C N Q 4$ variants are present in a 


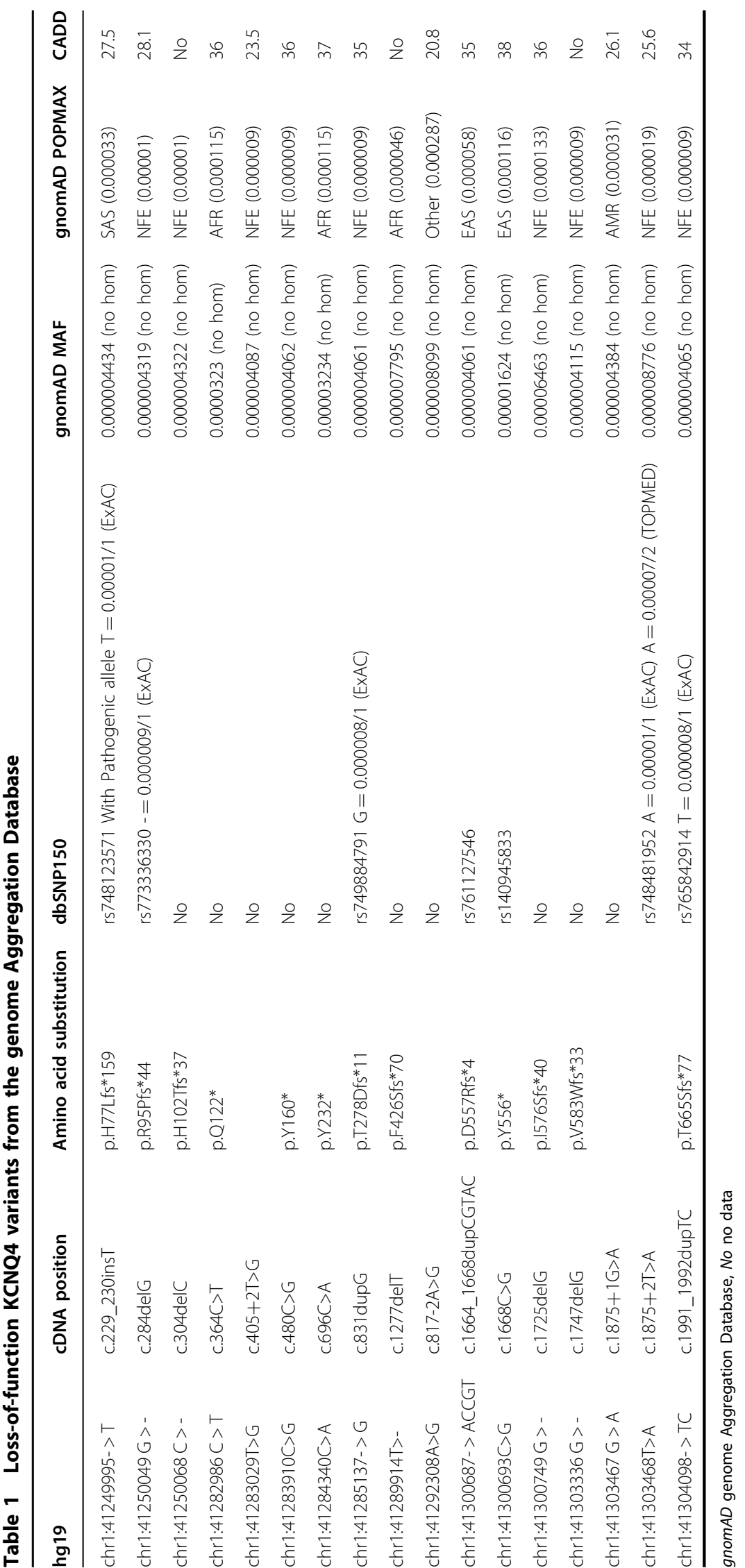




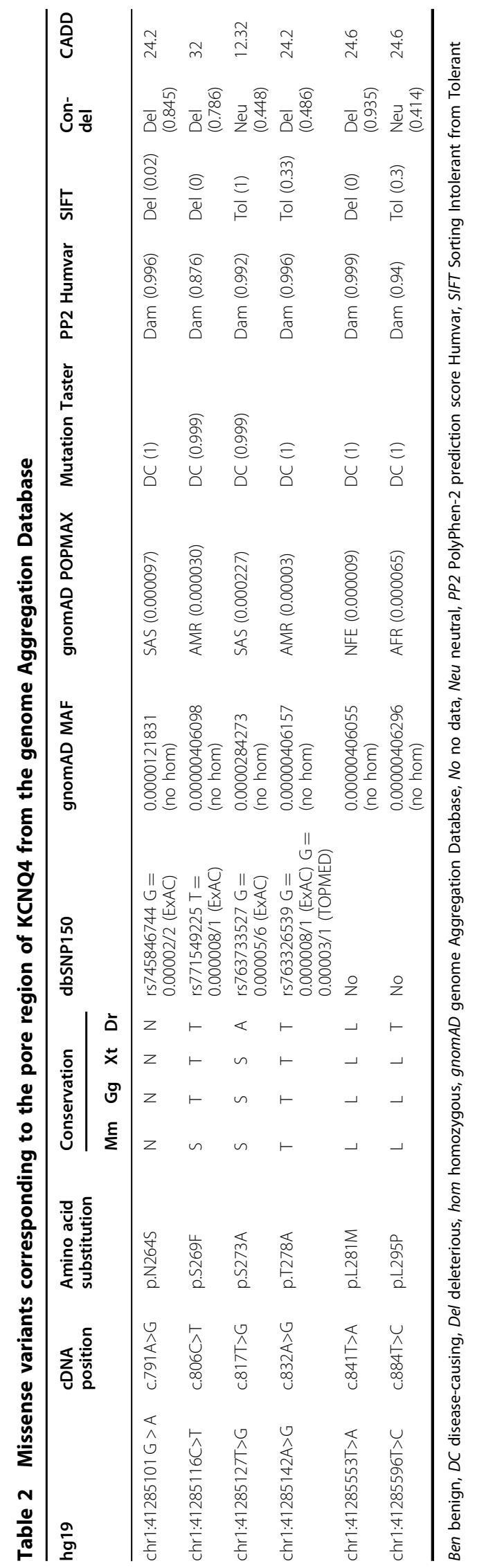

heterozygous state and exhibit a dominant-negative effect on functional WT channels, thereby decreasing potassium currents $^{12,23}$, we also examined whether six missense KCNQ4 variants around the pore region reported in gnomAD had a dominant-negative effect. We measured potassium currents in $\mathrm{CHO}$ cells cotransfected with WT and mutants (1:1 ratio) to mimic the heterozygous condition, and the results show that all six variants exerted dominant-negative inhibitory effects on WT KCNQ4mediated currents (Fig. 3d, e, and Supplementary Fig. 3).

\section{Rescue of potassium currents produced by KCNQ4 variants by retigabine}

Retigabine was developed as an anticonvulsant to treat epilepsy, acting primarily as a potassium channel (including KCNQ4) opener $^{24}$. Retigabine increased KCNQ4-mediated whole-cell current by more than 2-fold (Fig. 4a). We examined whether the impaired conductance of $\mathrm{KCNQ} 4$ variants could be recovered by retigabine. As shown in Fig. 4b, the activity of p.S273A, p. T278A, p.L281M, p.L295P, and p.R433W was rescued by retigabine, whereas that of p.N264S, p.S269F, and p. W276S was not. Therefore, variants showing reduced but still-present voltage-activated currents were rescued by retigabine to almost WT levels, whereas variants with almost null potassium currents were obviously nonrescuable (Fig. $4 \mathrm{~b}$ ). We also examined the effect of retigabine on DFNA2-associated $K C N Q 4$ variants reported in HGMD and measured ion conductance using the thallium-sensitive fluorescent dye FluxOR. Variants p. L47P, p.F182L, p.L281M, p.P291S, p.L285P, p.V672M, and p.S680F were rescued by retigabine (Fig. 4c), indicating that only some DFNA2-associated $K C N Q 4$ variants are rescued by retigabine. Interestingly, even though the variants occurred at the same amino acid residue, p.L281S did not respond to retigabine, whereas p.L281M was rescued by the drug (Fig. 4c).

To examine the effects of the variants at the protein level, we performed structural modeling of WT and variant KCNQ4 proteins based on the cryo-EM structure of Xenopus KCNQ1 ${ }^{25}$. The p.W276S variant was predicted to decrease the stability of KCNQ4 by three prediction programs (Supplementary Fig. 4). Six missense variants around the pore region were also predicted to have a destabilizing effect by at least one program (Fig. S4). However, the structural prediction results did not correlate with the reduction in current mediated by the variant KCNQ4 proteins, nor did they provide insight into the differences in the rescue of voltage-gated channel activity by retigabine among the KCNQ4 variants.

\section{Discussion}

Pathogenic $K C N Q 4$ variants cause progressive hearing loss inherited in an autosomal dominant manner 


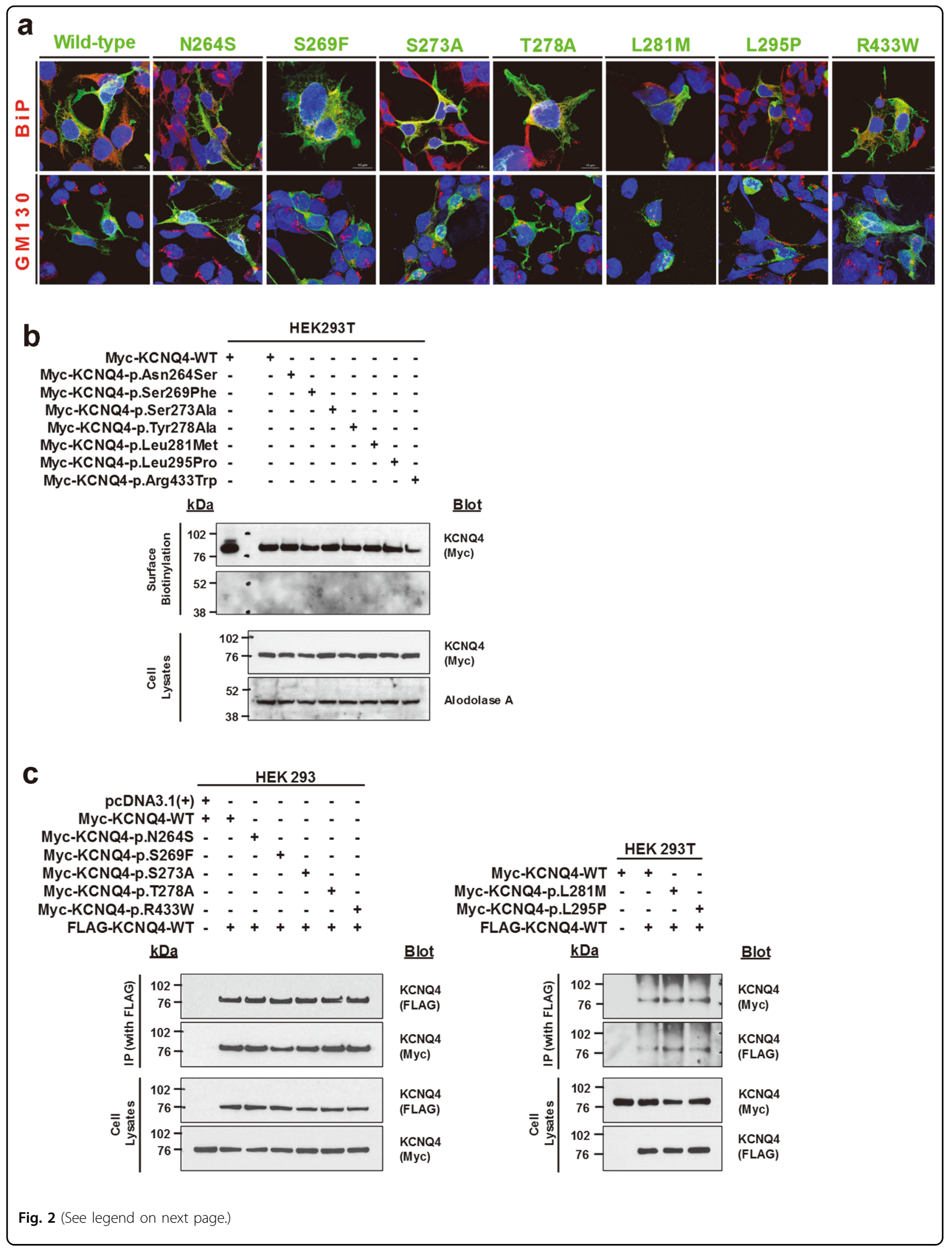


(see figure on previous page)

Fig. 2 Effects of KCNQ4 variants on protein expression on the cell surface and on subunit interaction. a Immunofluorescence of wild-type (WT) and variant KCNQ4 proteins in HEK 293 cells. Cells were immunostained with anti-Myc, anti-BiP, and anti-GOLGB1 antibodies. Nuclei were stained with DAPI. BiP and GOLGB1 indicate the endoplasmic reticulum and Golgi apparatus, respectively. All KCNQ4 variant proteins were observed on the plasma membrane. $\mathbf{b}$ Cell surface biotinylation. Proteins on the plasma membrane were labeled with biotin, isolated with avidin beads, and assessed by western blotting. Surface expression of KCNQ4 variant proteins was similar to that of the WT protein. c Coimmunoprecipitation. Cells were cotransfected with FLAG-tagged WT KCNQ4 and Myc-tagged WT or mutant KCNQ4 clones. After transfection (36 h), whole-cell lysates were subjected to immunoprecipitation using anti-FLAG beads and immunoblotted. All KCNQ4 variant proteins interacted with the WT protein

(DFNA2) ${ }^{3,10}$. Although slowly progressive hearing loss is not fully attributable to the degeneration of outer hair cells in the cochlea, the main pathomechanism is the impaired potassium recycling in the endolymph, which consequently changes the nature of its electrolyte milieu and debilitates the endocochlear potential ${ }^{26,27}$. In mice, the mechanism of hearing loss is not haploinsufficiency but a dominant-negative effect of the mutant KCNQ4 because heterozygous knockout mice do not show any hearing $\operatorname{loss}^{27}$. Therefore, most mutations identified in humans are thought to have a dominant-negative effect.

The majority of variants linked to DFNA2 are located around the pore region of the protein encoded by the KCNQ4 gene, which is responsible for the ion selectivity of the channel ${ }^{12,28}$. KCNQ4 mediates an M-like potassium current in the outer hair cells and type I vestibular hair cells $^{3,29}$. The M-like potassium conductance in these cells is dominant-negatively abolished when the pore mutant $\mathrm{p}$. G285S was transfected into the cells ${ }^{29}$. These findings indicate that the main pathomechanism of DFNA2 resulting from mutations in amino acid residues around the pore region of KCNQ4 is the dominant-negative loss of potassium current in the outer hair cells. Therefore, people with KCNQ4 variants with impaired potassium current are strong candidates for progressive hearing loss linked to DFNA2.

In this regard, we examined all missense variants changing residues around the pore region in KCNQ4 from public databases, including gnom $A D$, and found six missense variants that are present in the general population at low frequency (Supplementary Fig. 1). Because the variants have not been reported as pathogenic in HGMD or ClinVar, they are generally considered variants of unknown significance. Interestingly, we found that the $K C N Q 4$ variants around the pore region produced a significantly impaired potassium current. Given that several mutations in KCNQ4 give rise to proteins with impaired trafficking, the reduced current might be attributable to reduced expression levels of the channel on the cell surface $^{4}$. However, in our results, the KCNQ4 variants around the pore region showed normal membrane expression levels. In addition, we demonstrated that these six missense $K C N Q 4$ variants from public databases can exert a dominant-negative effect. This raises the possibility that individuals carrying these variants are at higher risk of developing late-onset or progressive hearing loss. In other words, the variants can be considered risk factors for late-onset or progressive hearing loss.

Based on MAFs (q) of six missense variants around the pore region of KCNQ4 reported in gnomAD, if we assume that these variants are in Hardy-Weinberg equilibrium $\left(\mathrm{p}^{2}+2 \mathrm{pq}+\mathrm{q}^{2}=1, \mathrm{p}+\mathrm{q}=1\right)$, we can calculate the frequencies of individuals who carry these variants in a homozygous or heterozygous state $\left(2 \mathrm{pq}+\mathrm{q}^{2}\right)$. Frequencies of heterozygous or homozygous individuals are estimated to be $0.0000244,0.0000081,0.0000569,0.0000081$, 0.0000081 , and 0.0000081 for p.N264S, p.S269F, p.S273A, p.T278A, p.L281M, and p.L295P, respectively. Their sum is 0.0001137 , which is $\sim 5.69 \%$ of the prevalence of hearing loss $(1 / 500=0.002)$. Therefore, KCNQ4 variants may contribute more than expected to hearing loss.

More than one hundred genes causing NSHL have been described (http://hereditaryhearingloss.org/). NSHL is very heterogeneously inherited. In particular, autosomal dominant hearing loss is mainly related to late-onset and progressive hearing loss; therefore, it is unclear whether young children or adults are affected by hearing loss. Those with mild or moderate hearing loss are likely considered healthy because high-frequency hearing loss is commonly undetected. Thus, it is plausible that sequencing data of people who have mild-to-moderate hearing loss or who are in a subclinical stage of progressive hearing loss are present in public databases. KCNQ4 variants attributable to ARHL or NIHL but not yet reported as pathogenic may also be included in public databases.

There are numerous ion channels in the inner ear, which are important for the maintenance of ion homeostasis and hearing function. Anion channels/transporters such as SLC26A4 and cation channels such as KCNQ1, KCNQE1, KCNQ4, KCNJ10, TRPV5, and TRPV6 are included in the list of deafness-related genes ${ }^{30}$. The results of the present study suggest that many unveiled pathologic variants may occur in these ion channel genes. Specifically, pore missense mutations are mainly attributable to progressive hearing loss. Further analyses are needed to uncover rare variants in these channel genes that confer a high risk for late-onset or progressive hearing loss.

$\mathrm{KCNQ}$ channels, particularly heteromeric $\mathrm{KCNQ} 2 / 3$, play a critical role in maintaining neuronal excitability by 

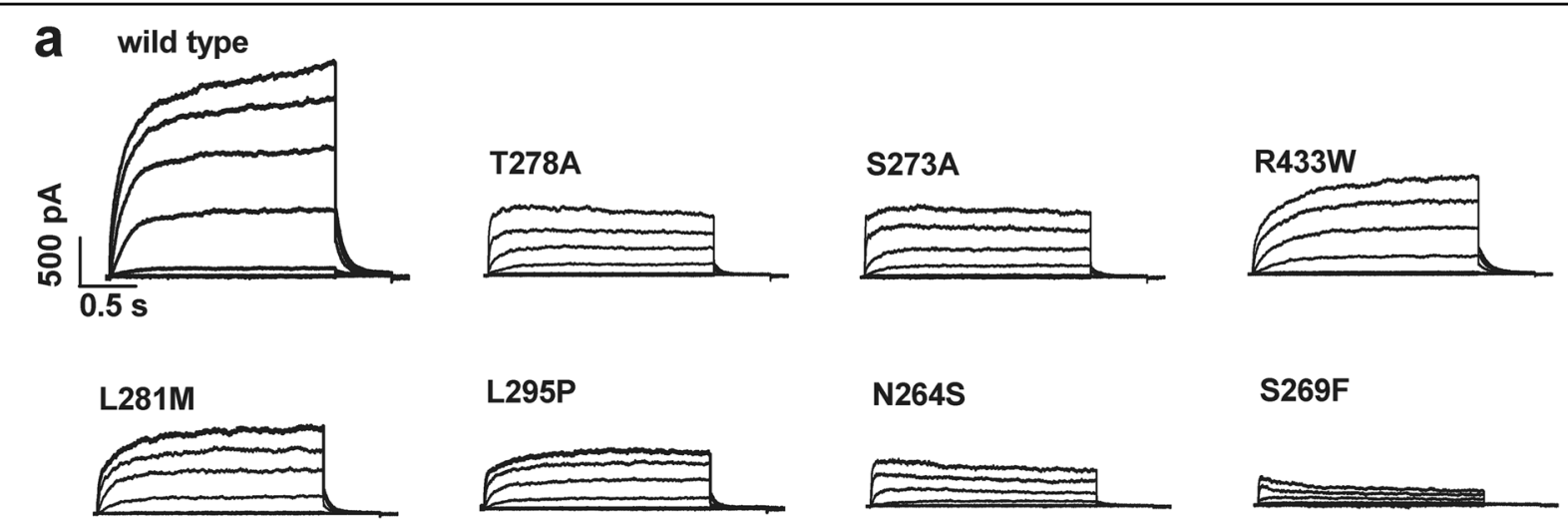

L295P

N264S

S269F
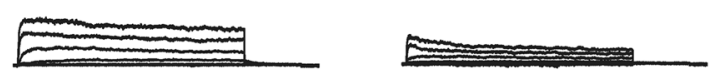

W276S

GFP

p.H455Q
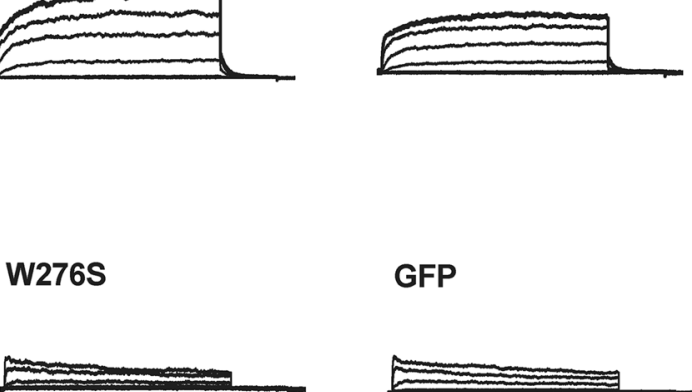

b
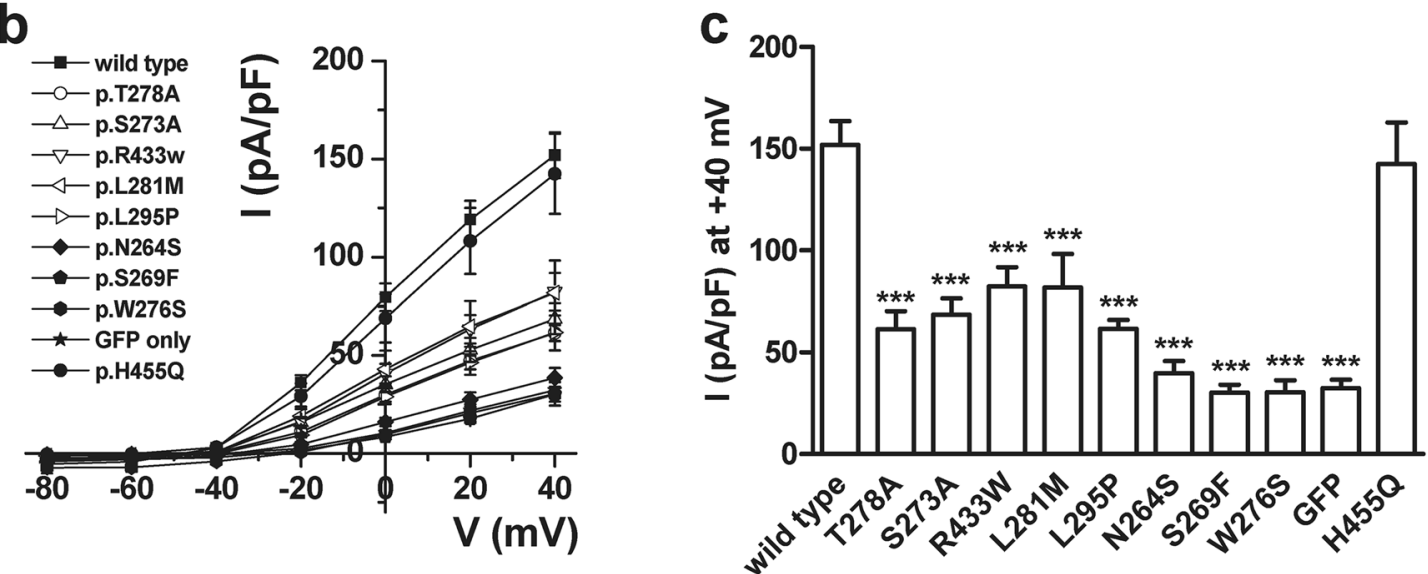

d

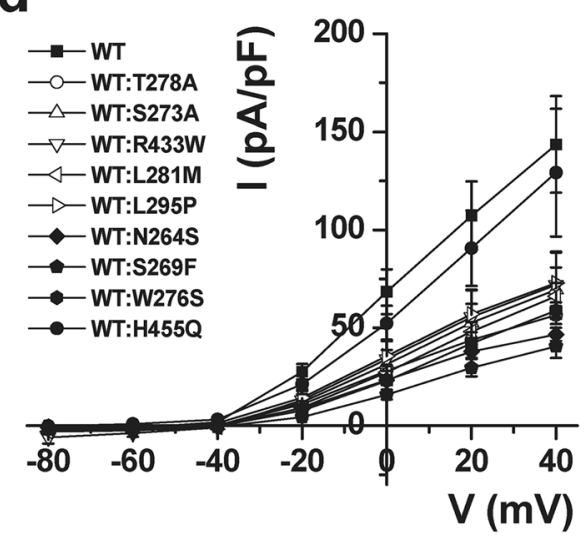

e

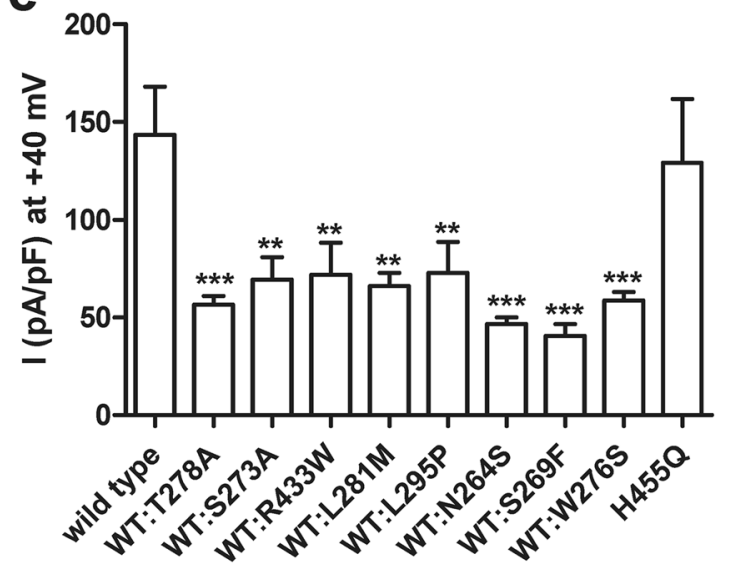

Fig. 3 (See legend on next page.) 
(see figure on previous page)

Fig. 3 Whole-cell currents produced by KCNQ4 mutants. a Representative whole-cell current traces recorded in CHO cells transfected with wildtype (WT) and mutant KCNQ4 with step pulses from $-80 \mathrm{mV}$ to $40 \mathrm{mV}$ in $20 \mathrm{mV}$ steps. b Mean current-voltage (I-V) relation curves obtained from steady-state currents elicited at each step pulse voltage. c Summary bar graph of steady-state currents elicited at $40 \mathrm{mV}$ normalized to membrane area (capacitance), obtained from whole-cell current recordings as presented in A. Averaged whole-cell current densities were 151.9 $\pm 11.6 \mathrm{pA} / \mathrm{pF}(n$ $=30), 61.5 \pm 8.8 \mathrm{pA} / \mathrm{pF}(n=19), 68.6 \pm 7.8 \mathrm{pA} / \mathrm{pF}(n=17), 81.8 \pm 16.4 \mathrm{pA} / \mathrm{pF}(n=12), 61.6 \pm 4.3 \mathrm{pA} / \mathrm{pF}(n=15), 82.3 \pm 9.5 \mathrm{pA} / \mathrm{pF}(n=19), 38.5 \pm 5 \mathrm{pA} /$ $\mathrm{pF}(n=16), 30.2 \pm 3.8 \mathrm{pA} / \mathrm{pF}(n=12), 30.4 \pm 5.9 \mathrm{pA} / \mathrm{pF}(n=8), 142.5 \pm 20.5 \mathrm{pA} / \mathrm{pF}(n=4)$, and $32.4 \pm 4.2 \mathrm{pA} / \mathrm{pF}(n=9)$ for WT, p.T278A, p.S273A, p. L281M, p.L295P, p.R433W, p.N264S, p.S269F, p.W276S, p.H455Q and green fluorescent protein only, respectively. Data represent the mean \pm SEM. ${ }^{* * *} P$ < 0.001 compared to WT. Statistical analysis was performed using one-way ANOVA with Bonferroni's multiple comparison. $\mathbf{d}$ Dominant-negative effects of mutants on KCNQ4-mediated currents were analyzed with a 1:1 ratio of WT and mutants KCNQ4 and step pulses from $-80 \mathrm{mV}$ to $40 \mathrm{mV}$ in $20 \mathrm{mV}$ steps. The mean I-V relation curve obtained from the steady-state current values elicited at each step pulse voltage is shown. e Summary bar graph of the steady-state currents elicited at $40 \mathrm{mV}$ normalized to capacitance. The averaged whole-cell current densities were $143.6 \pm 24.5 \mathrm{pA} / \mathrm{pF}(n$ =7), $56.6 \pm 4.5 \mathrm{pA} / \mathrm{pF}(n=11), 69.4 \pm 1.1 \mathrm{pA} / \mathrm{pF}(n=9), 72 \pm 16.4 \mathrm{pA} / \mathrm{pF}(n=12), 66.1 \pm 6.7 \mathrm{pA} / \mathrm{pF}(n=10), 72.9 \pm 15.9 \mathrm{pA} / \mathrm{pF}(n=10), 46.7 \pm 3.4 \mathrm{pA} /$ $\mathrm{pF}(n=9), 40.6 \pm 6.04 \mathrm{pA} / \mathrm{pF}(n=9), 58.8 \pm 4.2 \mathrm{pA} / \mathrm{pF}(n=10)$, and $129.2 \pm 32.5 \mathrm{pA} / \mathrm{pF}(n=4)$ for WT, p.T278A, p.S273A, p.R433W p.L281M, p.L295P, p. N264S, p.S269F, p.W276S, and p.H455Q, respectively. The red line is the predicted current density if there is no dominant-negative effect. Data represent the mean \pm SEM. ${ }^{* *} P<0.01,{ }^{* * *} P<0.001$ compared to the WT. Statistical analysis was performed using one-way ANOVA with Bonferroni's multiple comparison

producing outward $\mathrm{K}^{+}$currents to induce membrane repolarization and hyperpolarization ${ }^{31}$. Reduction in KCNQ2/3 channel activity causes neuronal disorders (hyperexcitability), such as epilepsy and tinnitus ${ }^{32}$. There have been many trials to develop therapies for these diseases in which KCNQ channels are activated to obtain neuronal hyperexcitability. In fact, retigabine, a small molecule that activates KCNQ2-5 channels, was approved as an anti-epileptic drug by the US Food and Drug Administration $^{33}$. Retigabine exerts its therapeutic effect by enhancing KCNQ channel activity through the shift of voltage-dependent activation to more negative voltages, resulting in a reduction in the excessive firing of neuronal cells ${ }^{34}$.

KCNQ4 channels expressed in the basolateral membrane of outer hair cells play a major role in maintaining the resting membrane potential. Therefore, impaired KCNQ4 channel activity caused by dysfunctional KCNQ4 mutants induces degeneration of outer hair cells by prolonged depolarization of the membrane potential, which further leads to progressive hearing $\operatorname{loss}^{35}$. In our studies, retigabine rescued the voltage-activated currents mediated by $\mathrm{KCNQ} 4$ pore mutants, suggesting that retigabine or other KCNQ activators may be useful in the therapeutics of genetic hearing loss. There is an unmet medical need for the treatment of inherited hearing loss caused by mutations in $K C N Q 4$. However, more in vitro studies and clinical trials are needed to determine the therapeutic effects of KCNQ activators on hearing loss.

Pore variants of KCNQ4 are known to be unresponsive to retigabine or zinc pyrithione, which is another KCNQ activator $^{36}$. Similarly, our results showed that the almost null potassium activity for the two mutants was not rescued by retigabine. However, it is noteworthy that four of the six pore variants of KCNQ4 with residual voltageactivated currents were activated by retigabine to almost WT levels. Retigabine increases channel function by stabilizing the open $\mathrm{K}^{+}$conducting form through binding to a single conserved tryptophan residue in the S5 domain (Supplementary Fig. 1) $)^{37,38}$. Our results show that retigabine did not enhance channel activity of variant KCNQ4 proteins with completely impaired channel function, whereas residual currents mediated by other variant KCNQ4 proteins were rescuable. Given that $\mathrm{KCNQ}$ activators increase KCNQ4 activity through alteration of channel conductance, it is still unclear how pore mutants are responsive to retigabine ${ }^{24,39}$. It is possible that retigabine induces a conformational change in the pore region. Further biophysical analyses are needed to clarify this issue.

Because KCNQ4 assembles into a homotetramer, 6.25\% of tetramers are formed of WT subunits only when a heterozygous KCNQ4 variant is present, which are potential therapeutic targets of retigabine if only the WT assembly was responsive to the drug. However, more potential retigabine targets would be available if the drug could also rescue the channel activity of pore mutant KCNQ4 as well as WT proteins.

In this context, we found that it may be possible to rescue channel activity by nonselective KCNQ activators, thereby preventing hearing deterioration in patients with DFNA2. However, retigabine has many systemic side effects, such as drowsiness, vertigo, and slurred speech, and thus has not been successful in the market ${ }^{32,40}$. To avoid these adverse effects, retigabine could be locally administered in the inner ear. Overall, the clinical value of this drug will be scientifically evaluated after further biophysical analysis is performed, and distinct in vivo study results are available.

In conclusion, we found that there are many unreported variants around the pore regions of KCNQ4, possibly associated with DFNA2, in public databases. Given that DFNA2 is one of the most common causes of ADNSHL, genetic testing of KCNQ4 is highly important. 


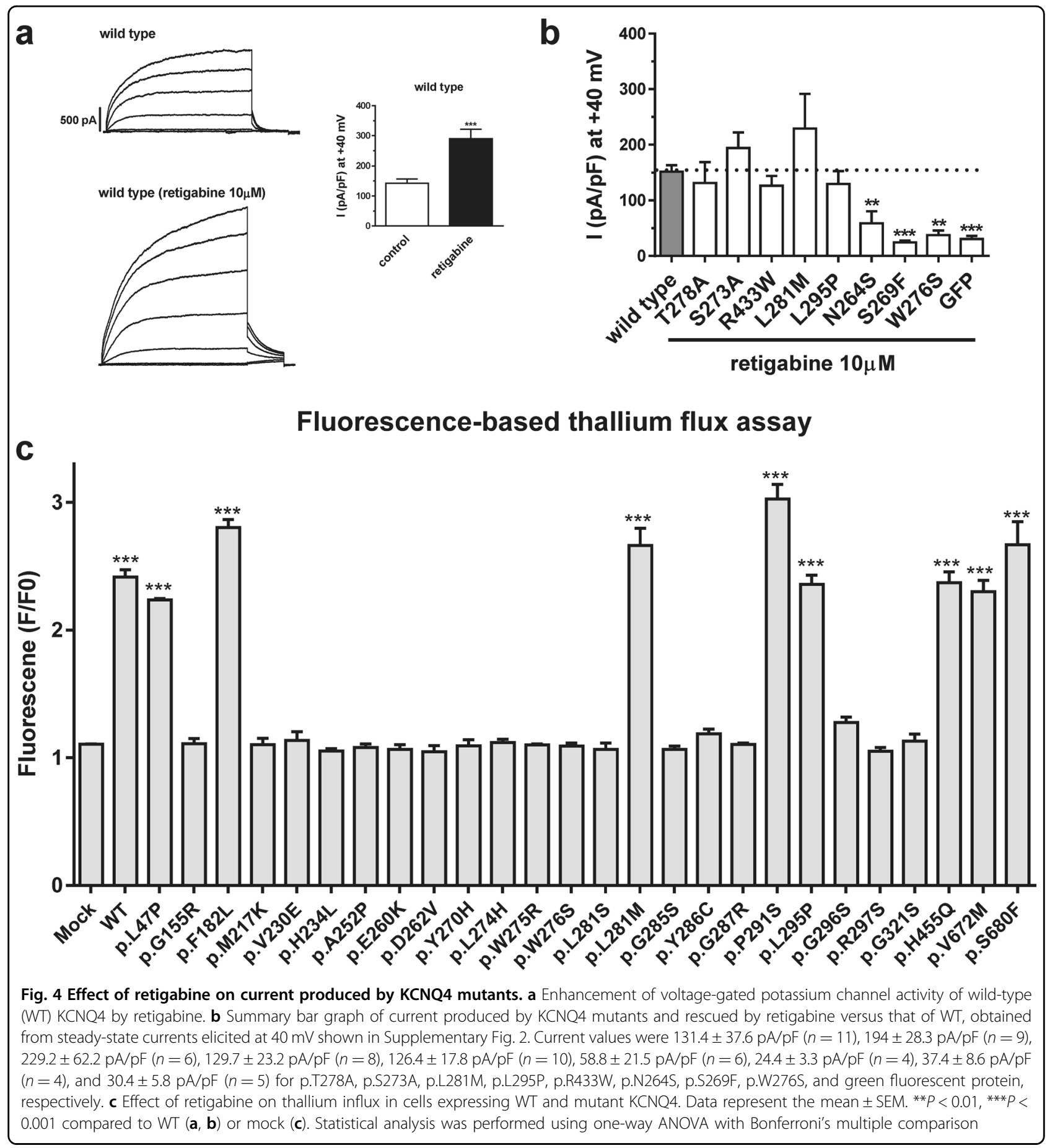

Furthermore, we identified several KCNQ4 pore variants potentially treatable with a small molecule, which would enable us to realize precision medicine in genetic hearing loss.

\section{Acknowledgements}

We thank Yonsei Advanced Imaging Center in cooperation with Carl Zeiss Microscopy. This study was supported by the Basic Science Research Program of the National Research Foundation of Korea (NRF) (MSIT; 2018R1A5A2025079 to H.Y.G. and 2016R1A2B4007268 to C.J.Y.), by the Ministry of Health \& Welfare, Republic of Korea (2017M3A9E8029714 to J.J.), and by the National Biobank of Korea, Centers for Disease Control and Prevention, Republic of Korea (4845301, 4851-302 and -307).

\section{Author details}

'Department of Otorhinolaryngology, Brain Korea 21 PLUS Project for Medical Sciences, Yonsei University College of Medicine, Seoul 03722, Korea. ${ }^{2}$ Department of Pharmacology, Brain Korea 21 PLUS Project for Medical Sciences, Yonsei University College of Medicine, Seoul 03722, Korea. ${ }^{3}$ Yonsei 
University College of Pharmacy, Incheon 21983, Korea. ${ }^{4}$ Yonsei University College of Medicine, Seoul 03722, Korea. ${ }^{5}$ Department of Dermatology, Seoul St. Mary's Hospital, College of Medicine, The Catholic University of Korea, Seoul 03722, Korea

\section{Conflict of interest}

The authors declare that they have no conflict of interest.

\section{Publisher's note}

Springer Nature remains neutral with regard to jurisdictional claims in published maps and institutional affiliations.

Supplementary information accompanies this paper at https://doi.org/ 10.1038/s12276-019-0300-9.

Received: 22 October 2018 Revised: 1 April 2019 Accepted: 6 May 2019. Published online: 21 August 2019

\section{References}

1. Van Hauwe, P., Coucke, P. \& Van Camp, G. The DFNA2 locus for hearing impairment: two genes regulating $\mathrm{K}+$ ion recycling in the inner ear. Brit $J$. Audio. 33, 285-289 (1999).

2. Wangemann, P. K+ cycling and the endocochlear potential. Hear Res. $\mathbf{1 6 5}$, 1-9 (2002).

3. Kubisch, C. et al. KCNQ4, a novel potassium channel expressed in sensory outer hair cells, is mutated in dominant deafness. Cell 96, 437-446 (1999).

4. Gao, Y., Yechikov, S., Vazquez, A. E., Chen, D. \& Nie, L. Impaired surface expression and conductance of the KCNQ4 channel lead to sensorineural hearing loss. J. Cell Mol. Med. 17, 889-900 (2013).

5. Naito, T. et al. Comprehensive genetic screening of KCNQ4 in a large autosomal dominant nonsyndromic hearing loss cohort: genotype-phenotype correlations and a founder mutation. PloS ONE 8, e63231 (2013).

6. Dominguez, L. M. \& Dodson, K. M. Genetics of hearing loss: focus on DFNA2. Appl Clin. Genet 5, 97-104 (2012).

7. Wang, H. et al. Targeted high-throughput sequencing identifies pathogenic mutations in KCNQ4 in two large Chinese families with autosomal dominant hearing loss. PloS ONE 9, e103133 (2014).

8. Kamada, F. et al. A novel KCNQ4 one-base deletion in a large pedigree with hearing loss: implication for the genotype-phenotype correlation. J. Hum. Genet 51, 455-460 (2006).

9. Kim, H. J., LV, P., Sihn, C. R. \& Yamoah, E. N. Cellular and molecular mechanisms of autosomal dominant form of progressive hearing loss, DFNA2. J. Biol. Chem. 286, 1517-1527 (2011).

10. Coucke, P. J. et al. Mutations in the KCNQ4 gene are responsible for autosomal dominant deafness in four DFNA2 families. Hum. Mol. Genet. 8, 1321-1328 (1999).

11. Talebizadeh, Z., Kelley, P. M., Askew, J. W., Beisel, K. W. \& Smith, S. D. Novel mutation in the KCNQ4 gene in a large kindred with dominant progressive hearing loss. Hum. Mutat. 14, 493-501 (1999).

12. Van Hauwe, P. et al. Mutations in the KCNQ4 K+ channel gene, responsible for autosomal dominant hearing loss, cluster in the channel pore region. Am. J. Med Genet 93, 184-187 (2000).

13. Van Camp, G. et al. A mutational hot spot in the KCNQ4 gene responsible for autosomal dominant hearing impairment. Hum. Mutat. 20, 15-19 (2002).

14. Hildebrand, M. S. et al. Audioprofile-directed screening identifies novel mutations in KCNQ4 causing hearing loss at the DFNA2 locus. Genet Med. 10, 797-804 (2008)

15. Arnett, J. et al. Autosomal dominant progressive sensorineural hearing loss due to a novel mutation in the KCNQ4 Gene. Arch. Otolanyngol. 137, 54-59 (2011).

16. Uchida, Y., Sugiura, S., Sone, M., Ueda, H. \& Nakashima, T. Progress and prospects in human genetic research into age-related hearing impairment. Biomed. Res. Int. 2014, 390601 (2014).
17. Van Eyken, E. et al. KCNQ4: a gene for age-related hearing impairment? Hum. Mutat. 27, 1007-1016 (2006).

18. Huang, Q. \& Tang, J. Age-related hearing loss or presbycusis. Eur. Arch. Otorhinolaryngol. 267, 1179-1191 (2010).

19. Van Laer, L. et al. The contribution of genes involved in potassium-recycling in the inner ear to noise-induced hearing loss. Hum. Mutat. 27, 786-795 (2006).

20. Gee, H. Y., Tang, B. L., Kim, K. H. \& Lee, M. G. Syntaxin 16 binds to cystic fibrosis transmembrane conductance regulator and regulates its membrane trafficking in epithelial cells. J. Biol. Chem. 285, 35519-35527 (2010).

21. Shin, D. H. et al. A recurrent mutation in KCNQ4 in Korean families with nonsyndromic hearing loss and rescue of the channel activity by KCNQ activators. Hum. Mutat. 40, 335-346 (2019).

22. Jung, J. et al. Whole-exome sequencing identifies two novel mutations in KCNQ4 in individuals with nonsyndromic hearing loss. Sci. Rep. 8, 16659 (2018).

23. Mencia, A. et al. A novel KCNQ4 pore-region mutation (p.G296S) causes deafness by impairing cell-surface channel expression. Hum. Genet. 123, 41-53 (2008).

24. Schroder, R. L. et al. KCNQ4 channel activation by BMS-204352 and retigabine. Neuropharmacology 40, 888-898 (2001).

25. Sun, J. \& MacKinnon, R. Cryo-EM structure of a KCNQ1/CaM complex reveals insights into congenital long QT syndrome. Cell 169, 1042-1050 (2017). e1049.

26. Beisel, K. W. et al. Differential expression of KCNQ4 in inner hair cells and sensory neurons is the basis of progressive high-frequency hearing loss. $J$. Neurosci. 25, 9285-9293 (2005).

27. Kharkovets, T. et al. Mice with altered KCNQ4 K+ channels implicate sensory outer hair cells in human progressive deafness. EMBO J. 25, 642-652 (2006).

28. Jentsch, T. J. Neuronal KCNQ potassium channels: physiology and role in disease. Nat. Rev. Neurosci. 1, 21-30 (2000).

29. Holt, J. R., Stauffer, E. A., Abraham, D. \& Geleoc, G. S. Dominant-negative inhibition of M-like potassium conductances in hair cells of the mouse inner ear. J. Neurosci. 27, 8940-8951 (2007).

30. Dror, A. A. \& Avraham, K. B. Hearing loss: mechanisms revealed by genetics and cell biology. Annu. Rev. Genet 43, 411-437 (2009).

31. Villa, C. \& Combi, R. Potassium channels and human epileptic phenotypes: an updated overview. Front Cell Neurosci. 10, 81 (2016).

32. Kalappa, B. I. et al. Potent KCNQ2/3-specific channel activator suppresses in vivo epileptic activity and prevents the development of tinnitus. J. Neurosci. 35, 8829-8842 (2015).

33. Tatulian, L., Delmas, P., Abogadie, F. C. \& Brown, D. A. Activation of expressed KCNQ potassium currents and native neuronal M-type potassium currents by the anti-convulsant drug retigabine. J. Neurosci. 21, 5535-5545 (2001).

34. Gunthorpe, M. J., Large, C. H. \& Sankar, R. The mechanism of action of retigabine (ezogabine), a first-in-class $\mathrm{K}+$ channel opener for the treatment of epilepsy. Epilepsia 53, 412-424 (2012).

35. Nie, L. KCNQ4 mutations associated with nonsyndromic progressive sensorineural hearing loss. Curr. Opin. Otolanyngol. Head. Neck Surg. 16, 441-444 (2008).

36. Leitner, M. G. et al. Restoration of ion channel function in deafness-causing KCNQ4 mutants by synthetic channel openers. Br. J. Pharmacol. 165, 2244-2259 (2012)

37. Schenzer, A. et al. Molecular determinants of KCNQ (Kv7) K+ channel sensitivity to the anticonvulsant retigabine. J. Neurosci. 25, 5051-5060 (2005).

38. Kim, R. Y. et al. Atomic basis for therapeutic activation of neuronal potassium channels. Nat. Commun. 6, 8116 (2015).

39. Xiong, Q., Sun, H. \& Li, M. Zinc pyrithione-mediated activation of voltage-gated KCNQ potassium channels rescues epileptogenic mutants. Nat. Chem. Biol. 3, 287-296 (2007).

40. Miceli, F., Soldovieri, M. V., Martire, M. \& Taglialatela, M. Molecular pharmacology and therapeutic potential of neuronal Kv7-modulating drugs. Curr. Opin. Pharm. 8, 65-74 (2008). 